\title{
Grosswohnsiedlungen in St. Petersburg zwischen sozialräumlicher Polarisierung und Persistenz
}

\author{
Isolde Brade, Carola Neugebauer, Leipzig, Konstantin \\ Axenov, St. Petersburg
}

\section{Einführung}

In nahezu allen europäischen Ländern prägen Grosswohnsiedlungen, die seit den 1970 er Jahren in Plattenbauweise errichtet wurden, das Bild der Städte in Ost wie in West. Im Osten entsprach der Bau von «Plattensiedlungen» dem Leitprinzip der sozialistischen Gesellschaft, nämlich die Schaffung gleichwertiger Wohn- und Lebensbedingungen und die «Einebnung der Unterschiede im städtischen Lebensniveau» (BURDACK \& RudOLPH 2001:262).

Mit der tief greifenden Systemtransformation nach 1990 veränderten sich in den postsozialistischen Ländern des mittleren und östlichen Europa die Rahmenbedingungen für sozialräumliche Differenzierungen grundlegend. Die Einführung marktwirtschaftlich orientierter Regulationsmechanismen, verbunden mit Möglichkeiten der Eigentumsbildung, der Wohnraumprivatisierung und der Herausbildung eines freien Wohnungsmarktes erweiterten das Wohnungsangebot innerhalb der Stadtregion. Die gesellschaftliche Wahrnehmung und Wertschätzung der sozialistischen Grosswohnsiedlungen als Wohnstandort sowie ihre soziostrukturellen Entwicklungstendenzen stehen daher nach wie vor im Fokus der Stadtforschung zum post-sozialistischen Raum (DIvINSKy 2004; DuRMANOV \& DubBEling 2004; KiCINSKi 2004; MilsteAD 2008; Musterd \& VAn Kempen 2007; Neugebauer et al. 2011; TsEnKova 2006; TuRKINGTON et al. 2004; VAN KEMPEN et al. 2005).

Die Befunde in der Fachliteratur dazu sind allerdings durchaus widersprüchlich. Einige Studien kommen zu der Schlussfolgerung, dass die Grosswohnsiedlungen in Mittel- und Osteuropa Problemgebiete in einer Abwärtsspirale sind, deren Bewohner langfristig sozialräumliche Marginalisierung erfahren werden (BeCKhoven \& VAN KeMPen 2006; Restate 2005; SzELENYI 1996; WASSENBERG 2004). Im Gegensatz dazu weisen andere Studien auf die hohe Zufriedenheit der Bewohner mit ihrer Wohnsituation hin und betonen die soziostrukturelle Heterogenität und Persistenz (Bernt \& Kabisch 2006; Burmeister 2009; Kovács \& Douglas 2004; Liebmann 2006; Neugebauer et al. 2011). Alice KAHL beobachtete beispielsweise seit Beginn der 1970 er Jahre in einer soziologischen
Längsschnittanalyse eine kontinuierlich hohe Wohnzufriedenheit der Bewohner in einer der grössten Plattensiedlungen der DDR, Leipzig-Grünau (KAHL 2003, weitergeführt von KABISCH \& GRossmanN 2010), und beleuchtet damit die Bewertung der Grosswohnsiedlung aus Sicht der Bewohner. Aus einer Aussenperspektive untersuchte AlMa von DER HAGEN-DEMSZKY eine Grosswohnsiedlung in Budapest hinsichtlich ihres Marktwertes und stellte für «Gazdagrét» durchaus mit Budaer Villenvierteln vergleichbare Quadratmeterpreise fest (HAGEN-DEMSZKY 2009). In der Literatur wurde die Differenziertheit der verschiedenen Typen sozialistischer Grosswohnsiedlungen mit ihren spezifischen Wohnqualitäten, tradierten symbolischen Konnotationen und soziostrukturellen Mustern jedoch bisher wenig reflektiert und diskutiert. Zudem fehlt den Studien oftmals die Einordnung ihrer Ergebnisse in den stadtregionalen Kontext, um die Rolle der untersuchten Grosswohnsiedlungen im Sozialgefüge der Stadt bewerten zu können.

Seit Mitte der 1990er Jahre erleben die Grosswohnsiedlungen in vielen postsozialistischen Städten bedeutsame bauliche Veränderungen: Neben der beginnenden Lückenbebauung in innerstädtischen Quartieren und der Errichtung von Einfamilienhaussiedlungen im Umland der Städte, konzentriert sich der privatwirtschaftlich getragene Wohnungsneubau vor allem auf den neuen Geschosswohnungsbau an den Stadträndern als Nachverdichtung vorhandener Grosswohnsiedlungen oder als Umsetzung bis 1990 unrealisierter, sozialistischer Wohnraumplanungen, indem bereits erschlossene Areale nun mit aktueller Wohnhochhausarchitektur bebaut werden (BRADE et al. 2009; Hirt 2008; Lenkeviciute 2006). Die neuen Geschosswohnungsbauten erscheinen dabei als «neue Grosswohnsiedlungen», auch wenn ihre Bauqualität, Bauplanung, und -durchführung nicht mit der der Grosswohnsiedlungen im Sozialismus gleichgesetzt werden kann. Der neue Geschosswohnungsbau entsteht als ein Segment des sich entwickelnden freien Wohnungsmarktes.

Wie die sozialistischen Grosswohnsiedlungen geniessen auch die neuen GWS im höheren Preissegment eine anhaltend grosse Nachfrage auf dem Wohnungsmarkt (RudolPH \& AxENov 2003). Allerdings fehlen bisher Untersuchungen, die die Rolle des neuen, stetig wachsenden Geschosswohnungsbaus für das sozialräumliche Gefüge der Stadtregion allgemein und für das der sozialistischen Grosswohnsiedlungen insbe- 
sondere hinterfragen. Infolgedessen bleibt das Bild zu den alten und neuen Grosswohnsiedlungen in postsozialistischen Stadtregionen bisher unvollständig und undifferenziert.

An diesen Defiziten setzt der folgende Beitrag an. Am Beispiel von St. Petersburg (Russland) wird ein differenzierender Blick in die «Schlafquartiere» der Stadt gewagt und folgenden Fragen nachgegangen:

- Welche Bedeutung haben heute die unterschiedlichen Typen sozialistischer und neuerer Grosswohnsiedlungen auf dem St. Petersburger Wohnungsmarkt?

- Welche symbolischen und soziostrukturellen Muster und Entwicklungstendenzen weisen diese heute auf?

- Zeichnen sich nach 1990 zwischen den Grosswohnsiedlungstypen wachsende, sozialräumliche Differenzierungen oder gar Polarisierungen $a b$ ? Wie sind die Grosswohnsiedlungen im sozialräumlichen Kontext der gesamten Stadtregion zu bewerten?

\section{Methodischer Ansatz}

Der Beitrag stützt sich auf Untersuchungen eines Forschungsprojektes der Deutschen Forschungsgemeinschaft (2007-2010) zu sozialräumlichen Differenzierungen in St. Petersburg. Methodische Merkmale sind die kritische Triangulation qualitativer und quantitativer sozialwissenschaftlicher Methoden (leitfadengestützte Experteninterviews z.B. mit Stadtverwaltung, Maklern, Wissenschaftlern, standardisierte Haushaltsbefragungen ( $\mathrm{N}=594)$, sekundärstatistische Datenanalysen) und der Mehr-Fallstudienansatz, mit Hilfe dessen fünf Wohnquartiere der Stadtregion St. Petersburg vergleichend untersucht wurden. $\mathrm{Zu}$ den untersuchten Wohnquartieren, in denen die Haushaltbefragungen durchgeführt wurden, gehört ein Wohnquartier, in dem jener Grosswohnsiedlungstyp überwiegt, der am meisten verbreitet ist, aber eine schlechte Bauqualität aufweist: die «Chruščëvka» $(\mathrm{N}=150)$. Das untersuchte Wohnquartier ist verkehrstechnisch schlecht angebunden und verfügt über keine attraktiven Versorgungs- und Dienstleistungseinrichtungen. Aus dem mittleren Preissegment wurde eine Plattensiedlung der «späten Sozialismusperiode» (Serie 137) untersucht ( $\mathrm{N}=146)$. Dieser Gebäudetyp entspricht etwa dem Standardtyp aller in den ehemals sozialistischen Ländern verbreiteten Wohnblöcke in Plattenbauweise. Als einer der bevorzugtesten Wohnungstypen unter den GWS wurde ein Wohnquartier des neuen Geschosswohnungsbaus nach 1995 ausgewählt ( $\mathrm{N}=146)$, das mit einem vielseitigen Angebot an modernen Versorgungs- und Dienstleistungseinrichtungen ausgestattet ist. Ausserdem wurden ein suburbanes Einfamilienhausgebiet im Norden der Stadtre- gion $(\mathrm{N}=55)$ sowie ein dicht bebautes Wohnquartier in der historischen Innenstadt untersucht $(\mathrm{N}=97)$.

Obwohl Langzeitstudien fehlen, können Aussagen zu symbolischen und soziostrukturellen Entwicklungstendenzen in den Grosswohnsiedlungen auf folgenden Grundlagen gemacht werden:

Entsprechende Aussagen können abgeleitet werden, indem die Merkmale der nach 1990 in ein untersuchtes Quartier zugezogenen Haushalte (Einkommen, Alter, Qualifikation, Haushaltstruktur u.ä.) mit denen der anderen untersuchten Wohnquartiere der Stadtregion verglichen werden. Diese intra-regionalen Kreuzvergleiche erlauben Aussagen dazu, inwieweit es einzelnen Wohnquartieren nach 1990 gelang, relativ zum stadtregionalen Kontext beispielsweise einkommensstärkere Haushalte anzuziehen und damit eine relative Tendenz der sozioökonomischen Aufwertung zu erfahren.

Neben den quantitativen wurden qualitative Analysen für Aussagen zu Dynamik und sozialräumlicher Differenzierung genutzt, die sich auf Experteninterviews, einschlägige Medienauswertungen und das Wissen der lange Jahre im Untersuchungsraum forschenden und beobachtenden Autoren zu den sozialräumlichen und symbolischen Mustern der Grosswohnsiedlungen stützen.

\section{Grosswohnsiedlungstypen (GWS) in St. Petersburg}

Nach dem zweiten Weltkrieg wurden in Leningrad (seit 1991 wieder St. Petersburg) zunächst alle Kräfte auf den Wiederaufbau des zerstörten historischen Stadtzentrums konzentriert. Wohnungsneubauten im neoklassizistischen «Stalinstil» in sehr guter Bauqualität säumten lediglich linienhaft einige Radialen bzw. punkthaft einige Plätze als städtische Dominanten. Sie wurden nach individuellen Projekten entwickelt, vermochten aber nicht annähernd das Problem des Wohnraumdefizits zu lösen.

Erst mit der Machtübernahme von Nikita Chruščèv und der Umorientierung der Wohnungspolitik von hohen Qualitätsansprüchen auf mehr Quantität, entstanden ab Ende der 1950er Jahre die ersten kompakt bebauten fünfgeschossigen Wohnsiedlungen in Leningrad, die sogenannten Chruščëvki. Die von der Regierung verkündete Aufgabe, im Verlaufe eines kürzest möglichen Zeitraumes jeder sowjetischen Familie eine eigene Wohnung zur Verfügung zu stellen, stellte die Stadtpolitiker von Leningrad vor eine komplizierte Aufgabe. Mehrere Industrialisierungswellen seit Ende der 1920er Jahre, die Zerstörungen durch zwei Weltkriege und den Bürgerkrieg (1918-1920) sowie die uneffektive Wohnungspolitik seit den 1930er Jahren hatten dazu 


\begin{tabular}{|c|c|c|c|}
\hline «Macht-Phasen» & $\begin{array}{l}\text { Wohnungs- } \\
\text { neubau, in }\end{array}$ & $\begin{array}{l}\text { Anteil am gesam- } \\
\text { ten Wohnungs- } \\
\text { neubau 1945-2005, } \\
\text { in Prozent }\end{array}$ & Merkmale \\
\hline $\begin{array}{l}1945-1955 \\
\text { «Stalin-Periode» }\end{array}$ & 6027 & 7,7 & $\begin{array}{l}\text { grosszügig geschnittener Wohnraum, gute } \\
\text { technische Ausstattung der Häuser, solide } \\
\text { Bauqualität, gut begrünte Innenhöfe }\end{array}$ \\
\hline $\begin{array}{l}\text { 1956- } 1965 \\
\text { «Chruščëv-Periode» } \\
\text { (Chruščëvki) }\end{array}$ & 14752 & 18,9 & $\begin{array}{l}\text { Geringe Wohnfläche, kleine Zimmer, ohne } \\
\text { Lift und Balkon, mangelnde Bauqualität, } \\
\text { minimale Schall- und Wärmeisolation, gut } \\
\text { begrünte Innenhöfe, inzwischen sehr hoher } \\
\text { Sanierungsbedarf, 5-geschossige Bebauung }\end{array}$ \\
\hline $\begin{array}{l}1966-1975 \\
\text { «frühe Brežnev- } \\
\text { Periode » }\end{array}$ & 21566 & 27,7 & $\begin{array}{l}\text { Grosszügige Freiflächen, begrünte Innen- } \\
\text { höfe, quartiersnahe Kinder- und Bildungs- } \\
\text { einrichtungen, inzwischen hoher Sanierungs- } \\
\text { bedarf, vorrangig 9-geschossige Bebauung }\end{array}$ \\
\hline $\begin{array}{l}1976-1985 \\
\text { «Später } \\
\text { Sozialismus» (Serie } \\
\text { 137) }\end{array}$ & 16916 & 21,7 & $\begin{array}{l}\text { Grosszügiger Wohnungsgrundriss, Balkon } \\
\text { oder Loggia, verzögerte infrastrukturelle } \\
\text { Ausstattung, inzwischen hoher Sanierungs- } \\
\text { bedarf, vorrangig 9- und 11-geschossige } \\
\text { Bebauung }\end{array}$ \\
\hline $\begin{array}{l}1986-1995 \\
\text { «Perestroika» }\end{array}$ & 7827 & 10.0 & $\begin{array}{l}\text { Verzögerte Wege- und Flächengestaltung, } \\
\text { ambulanter Einzelhandel, ungünstiger } \\
\text { öffentlicher Personennahverkehr, vorrangig } \\
\text { 11-geschossige Bebauung und höher }\end{array}$ \\
\hline $\begin{array}{l}1996-2005 \\
\text { «Transformations- } \\
\text { Periode» (Ge- } \\
\text { schosswohnungs- } \\
\text { bau nach 1995) }\end{array}$ & 10857 & 13,9 & $\begin{array}{l}\text { Gute Bauqualität, wenig Grün- und Frei- } \\
\text { flächen, dichte Bebauung, vorrangig } \\
\text { über 11-geschossige Bebauung }\end{array}$ \\
\hline Gesamt & 77945 & 100,0 & \\
\hline
\end{tabular}

Tab. 1: Wohnungsneubau während der politischen «Macht-Phasen»

Housing construction during the different periods of government

Construction de logements pendant les différentes phases de gouvernements

Quelle: SAnkt-Peterburg 1703-2003 (2004); SAnKT-Peterburg 2005 (2006)

geführt, dass in der Stadt nicht nur ein Wohnungsdefizit, sondern grosse Wohnungsnot herrschte.

Um das eklatante Wohnungsdefizit abzubauen, wurden ab Ende der 1960er Jahre Grosswohnsiedlungen vor allem in die Höhe und in einem bis dahin nicht praktizierten Ausmass gebaut. Diese «Mikrorajony» wurden für jeweils $100^{\prime} 000$ bis $200^{\prime} 000$ Einwohner mit 9- bis 11-geschossigen Wohnhäusern und den entsprechenden Grundversorgungseinrichtungen für Bildung, Gesundheit, Einzelhandel konzipiert.
Die in sowjetischer Zeit definierte Funktion der Grosswohnsiedlungen am Stadtrand als «Schlafstädte» ist dabei bis in die jüngste Zeit erhalten geblieben, auch wenn inzwischen Dienstleistungsund Freizeitzentren über den Grundbedarf hinaus angesiedelt wurden.

Nach wie vor lebt der grösste Teil der Bevölkerung in diesen Grosswohnsiedlungen der sowjetischen Bauphase. In St. Petersburg betraf das 2007 etwa $68 \%$ der Einwohner (AKsËNOv 2008). 
separate Wohnungen in sanierten Häusern im historischen Zentrum

separate Wohnungen in «Stalin»-Häusem

separate Wohnungen in unsanierten Häusern im historischen Zentrum (eigene Sanierung)

separate Wohnungen in Häusern mit genossenschaftlichem Eigentum (Plattenbau)

separate Wohnungen in Ziegelbau-Häusern, gebaut Ende der 1970 - Ende der 1980er Jahre

separate Wohnungen in Grosswohnsiedlungen der «späten Sozialismusperiode»

separate Wohnungen in Grossw ohnsiedlungen der «frühen Brežnev-Periode», 1970er Jahre

separate Wohnungen in Siedlungen der Chruščëvk-Ära, Ende 1950 - Ende 1960er Jahre (Chruščëvki)

Zimmer in einer Kommunalka

Zimmer in Arbeiter- und Studentenwohnheimen

Abb. 1: Hierarchie der Präferenzen für Wohnungstypen (in absteigender Rangfolge)

Hierarchy of preferences regarding different dwelling types

Hiérarchie des préférences concernant différents types de logements

Quelle: eigene Erhebungen (2007)

Seit Mitte der 1990er Jahre entstanden die «neuen Grosswohnsiedlungen» fast ausschliesslich durch privatwirtschaftliche Unternehmen. Die Wohnbauten mit neun bis mehr als 20 Etagen, zumeist in kombinierter Ziegel-/Plattenbauweise, haben sich als stark nachgefragter Wohnungsteilmarkt etabliert, obwohl hochwertig sanierte innerstädtische Wohnungen als eines der ersten Segmente auf dem neu herausgebildeten Wohnungsmarkt erschienen und im suburbanen Raum Einfamilienhaussiedlungen entstanden.

\section{Sozialräumliche Segregation auf «sowjetisch»}

Während der sozialistischen Stadtentwicklung hatte Segregation als Projektion sozialer Ungleichheit im
Raum keine annähernd vergleichbare Bedeutung wie in den westlichen Grossstädten (STEINFüHrer 2001). Aufgrund unterschiedlichen Zugangs $\mathrm{zu}$ bestimmten Privilegiensystemen sind dennoch Ansätze einer gesteuerten Segregation nachweisbar. Das Instrument dafür war die zentral geregelte wohnungspolitische Vergabe- und Zuteilungspraxis.

In St. Petersburg wurden daher die städtischen Wohnquartiere von den Bewohnern durchaus unterschiedlich bewertet und in einem Imagegefüge hierarchisiert. Wichtige Bewertungskriterien waren die Ausstattung des Wohnquartiers mit Versorgungseinrichtungen, der Wohnungstyp und die Eigentumsform (kommunales, genossenschaftliches oder Betriebseigentum). Die Lage spielte eine untergeordnete Rolle (Axenov, BRADE \& BondarchuK 2006). Bedeutsam war die Wohnungspolitik der jeweiligen «Machtphasen» für die Quartierserbauung (Tab. 1).

Die verschiedenen Typen sozialistischer Grosswohnsiedlungen bekamen danach in der gesellschaftlichen Wahrnehmung ungleiche Images (symbolische Konnotationen) zugewiesen. In Abbildung 1 ist beispielhaft die Präferenzhierarchie bezüglich der stadtregionalen Wohnungstypen in St. Petersburg im Sozialismus dargestellt.

Entscheidend für die sozialräumlichen Muster in der Stadtregion waren der Zugang zu Bildung, Kultur und Wohnraum, und weniger das Einkommen der Personen. In Tabelle 2 sind die Muster der sozialistischen sozialräumlichen Differenzierung rekonstruiert. Dabei wird deutlich, dass die sozialistischen Grosswohnsiedlungen keineswegs Wohnort der «sowjetischen Eliten» waren, sondern insbesondere der Mittelschicht.

\section{Grosswohnsiedlungen heute: sozialräumliche Muster und Entwicklungstendenzen}

Mit der Liberalisierung der Wohnungspolitik seit den 1990er Jahren und den oben erwähnten neuen Wohnungsmarktsegmenten haben sich die Präferenzen sowohl aus Sicht von Aussenstehenden (Wohnideale der Bewohner der Gesamtstadt, Abb. 2) als auch aus Sicht der jeweiligen Quartiersbewohner selbst (Abb. 2 und Abb. 3) gewandelt.

In beiden Abbildungen wird deutlich, dass das Einfamilienhaus im Grünen sowohl innerhalb der gesamtstädtischen als auch innerhalb der Einwohnerschaft einzelner Wohnquartiere dem bevorzugten Wohnideal entspricht. Innerstädtisches Wohnen sowie das Wohnen in den nach 1995 gebauten Hochhäusern der Grosswohnsiedlungen stehen den Präferenzen für suburbanes Wohnen deutlich nach. Mit Blick auf die 


\begin{tabular}{|c|c|c|}
\hline Wohnformen & Merkmale der Bewohnerschaft & Hintergründe \\
\hline $\begin{array}{l}\text { Quartiere im historischen } \\
\text { Stadtzentrum und «Stalin»- } \\
\text { Wohnkomplexe, entlang } \\
\text { der südlichen Ausfall- } \\
\text { strasse nach Moskau }\end{array}$ & $\begin{array}{l}\text { v.a. Wohnort von Vertretern der } \\
\text { mittleren und oberen } \\
\text { Leitungsebene aus Partei, } \\
\text { Administration, Militär, } \\
\text { Wirtschaft, Kultur- und } \\
\text { Kunstschaffende }\end{array}$ & $\begin{array}{l}\text { Einziges Beispiel dafür, dass auch die } \\
\text { Lage eine gewisse Rolle bei der } \\
\text { sozialräumlichen Segregation spielte. } \\
\text { Konzentration um Arbeitsstätten (z.B. } \\
\text { Smolny-Kloster als Sitz der Administra- } \\
\text { tion: Beschäftigte in Administration } \\
\text { und Parteiapparat; Stadtteile mit } \\
\text { Hochschulen und Theater: } \\
\text { akademische und Kulturangestellte) }\end{array}$ \\
\hline $\begin{array}{l}\text { Genossenschaftlicher } \\
\text { Wohnungsbau, vor allem } \\
\text { Grosswohnsiedlungen der } \\
\text { 1970-80 Jahre }\end{array}$ & $\begin{array}{l}\text { v.a. Wohnort von Vertretern der } \\
\text { «technischen Intelligenz»: } \\
\text { Ingenieure, mittleres } \\
\text { Verwaltungspersonal der } \\
\text { Grossbetriebe und } \\
\text { Forschungsinstitute }\end{array}$ & $\begin{array}{l}\text { Entstanden infolge der } 1962 \text { verab- } \\
\text { schiedeten Verordnung des ZK der } \\
\text { KPdSU und des Ministerrates der } \\
\text { UdSSR «Über den individuellen und } \\
\text { kooperativen Wohnungsbau»; } \\
\text { Bauumfang war eingeschränkt und der } \\
\text { Zugang zu Kooperativen staatlich } \\
\text { reguliert. Anteil } 1990 \text { am Wohnungs- } \\
\text { bestand: } 13,5 \% \text {. }\end{array}$ \\
\hline $\begin{array}{l}\text { Betriebswohnsiedlungen } \\
\text { der 1960-1980er Jahre, } \\
\text { Grosswohnsiedlungen, u.a. } \\
\text { Chruščëvki }\end{array}$ & $\begin{array}{l}\text { v.a. Wohnort von Arbeitern, } \\
\text { Angestellten und ingenieur- } \\
\text { technischem Personal }\end{array}$ & $\begin{array}{l}\text { Grossbetriebe errichteten eigene, vom } \\
\text { jeweiligen Industrieministerium } \\
\text { finanzierte Wohnsiedlungen oder } \\
\text { beteiligten sich finanziell am kommu- } \\
\text { nalen Wohnungsbau und erhielten } \\
\text { entsprechenden Anteil an Wohnungen } \\
\text { zur eigenen Verteilung. Anteil } 1990 \text { am } \\
\text { Wohnungsbestand: } 9,9 \% \text {. }\end{array}$ \\
\hline $\begin{array}{l}\text { Wohnquartiere mit einer } \\
\text { hohen Konzentration von } \\
\text { Wohnheimen, vor allem in } \\
\text { Chruščëvki }\end{array}$ & $\begin{array}{l}\text { Wohnort von Arbeitern, } \\
\text { Wochenendpendlern und } \\
\text { Studierenden }\end{array}$ & $\begin{array}{l}\text { Entstanden aufgrund grosser } \\
\text { Wohnungsnot, Konzentration an } \\
\text { einigen wenigen Standorten in Betriebs- } \\
\text { nähe; in den } 1970 \text { er Jahren lebten darin } \\
\text { mehr als } 300^{\circ} 000 \text { Einwohner. }\end{array}$ \\
\hline
\end{tabular}

Tab. 2: Merkmale sozialistischer sozialräumlicher Differenzierung

Features of socialist socio-spatial differentiation

Caractéristiques de la différenciation socio-spatiale socialiste

Quelle: RudolPH \& Axenov 2003; Predloženija po koordinacii Genplana 1980; Spravočnik o domovom fonde Leningrada 1990

sozialistischen Grosswohnsiedlungen zeigt sich, dass diese im Vergleich zu den neuen Wohnungsmarktsegmenten «suburbanes Wohnen», «innerstädtischer Wohnungsneubau» sowie «neuer Geschosswohnungsbau am Stadtrand» aktuell deutlich weniger stark präferiert werden. Die in Abbildung 1 skizzierte Präferenzhierarchie hat sich demnach zu Ungunsten der sozialistischen Grosswohnsiedlungen verändert: Sie sind in der Präferenzhierarchie zu den neuen
Wohnungsmarktsegmenten relativ gesunken und damit symbolisch abgewertet worden, ohne dass dies gleichzeitig hohe Unzufriedenheit und Ablehnung der sowjetischen GWS bei den Bewohnern selbst bedeuten würde.

Hinsichtlich unterschiedlichen Typen sozialistischer Grosswohnsiedlungen verdeutlicht Abbildung 3 jedoch auch Persistenzen bzw. Ähnlichkeiten im 


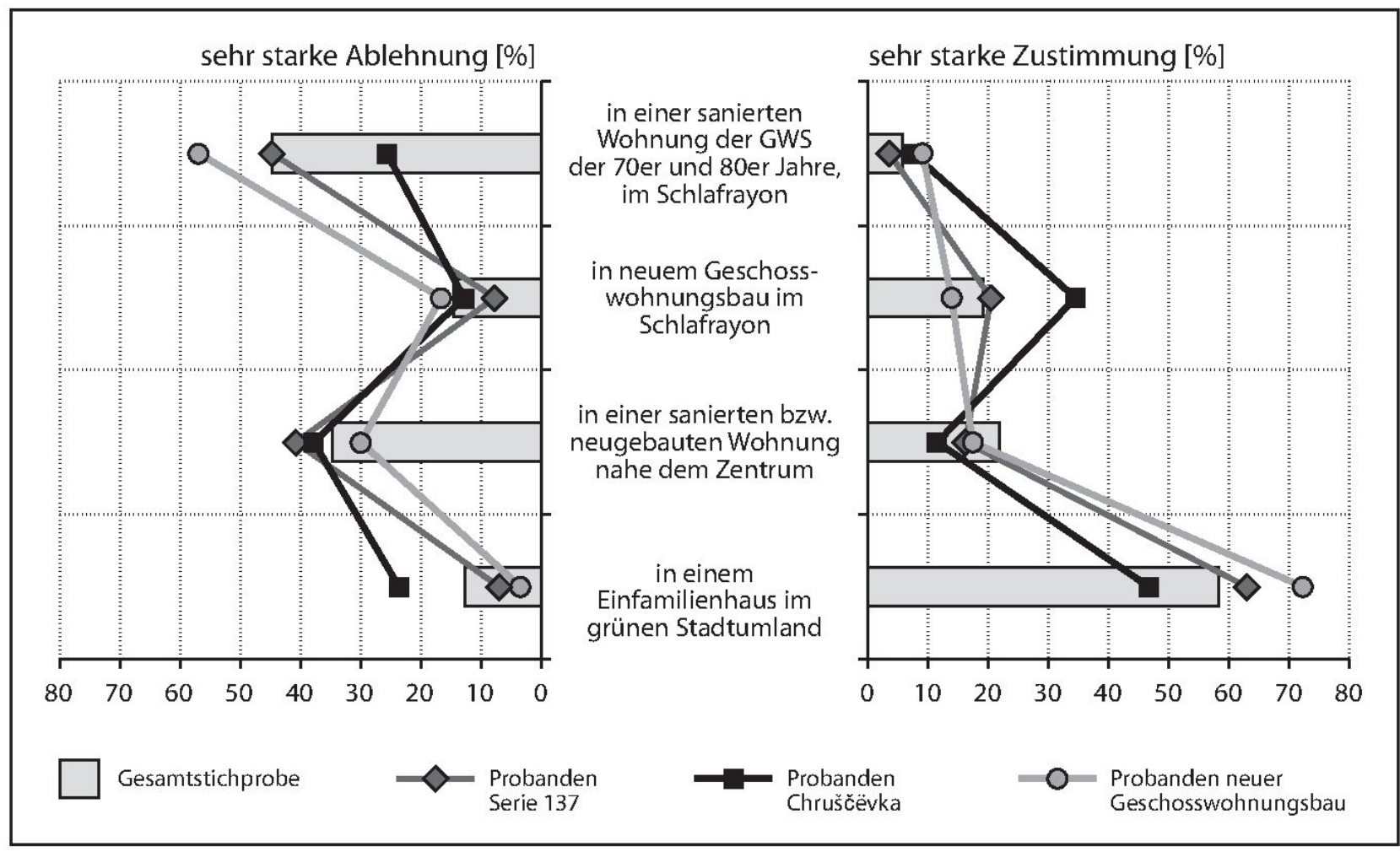

Abb. 2: Aktuelle Wohnideale, differenziert für die Respondenten der Grosswohnsiedlungen und der Gesamtstichprobe (in Prozent)

Current housing ideals: the opinions of large scale housing dwellers in comparison to the whole sample Idéaux résidentiels actuels: avis des habitants des grands ensembles par rapport au total de l'échantillon Quelle: eigene Erhebungen (2007); Entwurf: Projekt MOE Wohnen; Graphik: A. MüLLER

Vergleich zur Zeit vor 1990 (Abb. 1). So hat sich die symbolische Rangordnung unter den verschiedenen sozialistischen GWS nicht verändert: die «Chruščëvki» heben sich vielmehr nach wie vor von dem Grosswohnsiedlungstyp des Spätsozialismus (Serie 137) ab und sind noch immer Wohnsiedlungen mit geringerem Image, in denen verhältnismässig wenige mit ihrer Wohnung Zufriedene leben, während die Grosswohnsiedlungen der Serie 137 nach wie vor eine mittlere Wertschätzung erfahren.

Vor dem Hintergrund dieser symbolischen Veränderungen als auch Persistenzen nach 1990 in der Akzeptanz der Grosswohnsiedlungen schliesst sich die Diskussion der soziostrukturellen Entwicklungstendenzen und Muster in den alten und neuen GWS an. Tabelle 3 enthält Aussagen, inwieweit die GWS nach 1990 von residentieller Mobilität betroffen waren. Es wird deutlich, dass die Serie 137 im Vergleich zu den anderen, vor 1990 errichteten Wohnquartieren den geringsten Zuzug nach 1990 hatte und die Bewohnerstrukturen relativ stabil blieben. Im Unterschied dazu zeigt sich für die «Chruščëvki» ebenso wie für das innerstädtische Wohnquartier eine höhere Fluktuation. Insgesamt fand jedoch seit 1990 in keinem der vor 1990 errichteten Wohnquartiere ein Bevölkerungsaustausch statt.

Abbildung 4 verdeutlicht, inwiefern der Zuzug nach 1990 die soziostrukturellen Positionen der untersuchten Grosswohnsiedlungen im Sozialraumgefüge der Stadt relativ verändert haben könnte. Die Befragten wurden gebeten, ihre familiäre Einkommenssituation selbst einzuschätzen und sich folgende Kategorien zuzuordnen:

- Das Geld reicht gerade aus, um Lebensmittel zu kaufen.

- Das Geld reicht für Lebensmittel und Kleidung. Der Kauf von längerfristigen Gütern wie z.B. Haushaltgeräte, Fernseher, Computer ist mit Schwierigkeiten verbunden.

- Wir können uns längerfristige Güter wie z.B. Haushaltsgeräte, Fernseher, Computer leisten, der Kauf eines PKWs ist mit Schwierigkeiten verbunden. 


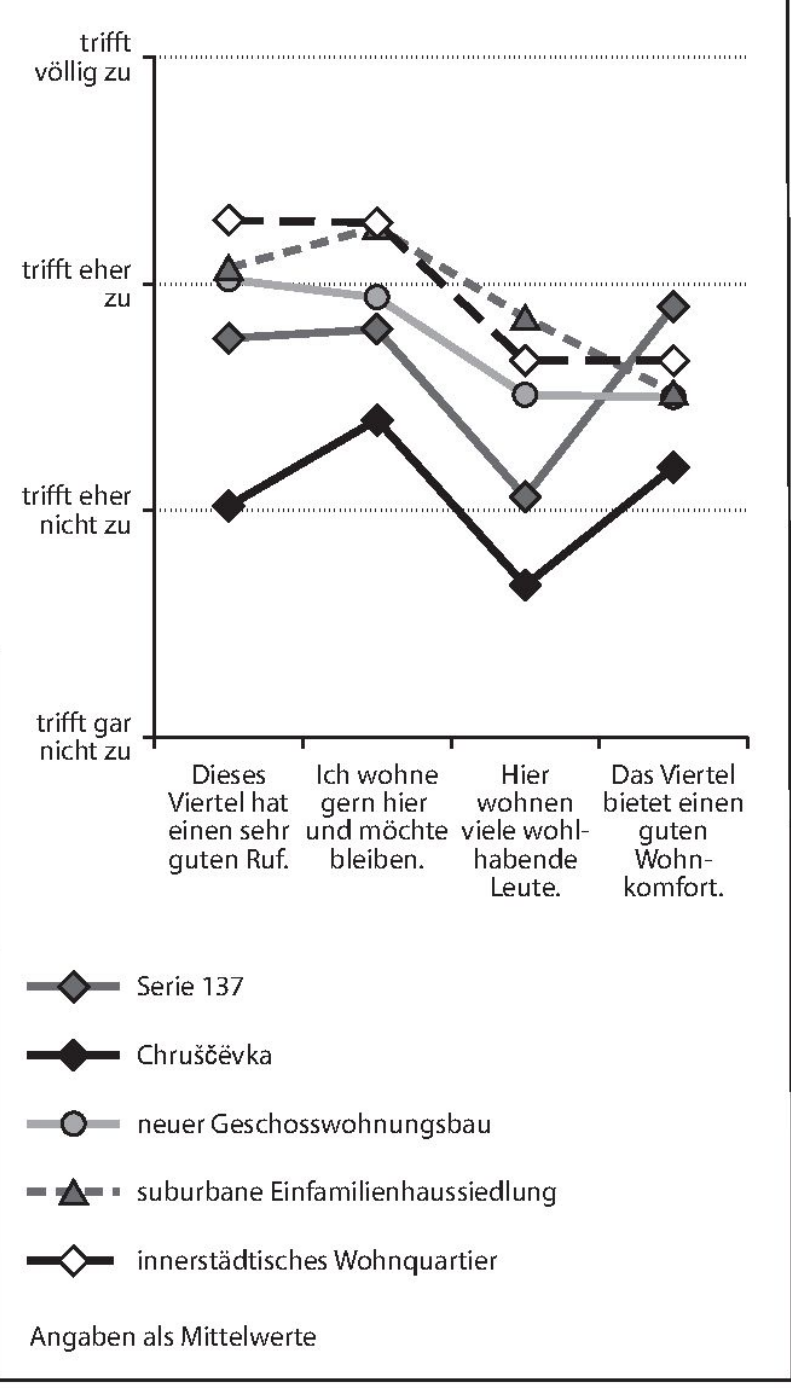

Abb. 3: Merkmale der Wohnquartiere und Wohnzufriedenheit der jeweiligen Quartiersbewohner Neighbourhood qualities and housing satisfaction of the respective neighbourhood dwellers

Caractéristiques du quartier et satisfaction résidentielle des habitants

Quelle: eigene Erhebung (2007); Entwurf: Projekt MOE Wohnen; Graphik: A. MÜLLER

- Wir können uns ein Auto leisten, aber wir können uns keine eigene Wohnung/Haus kaufen.

- Wir können uns alles ohne Schwierigkeiten leisten.

Diese subjektiven Einschätzungen der Befragten erlauben, die «gefühlten» Handlungsspielräume der Befragten $\mathrm{zu}$ verstehen. Die Abfrage des absoluten Haushalteinkommens hätte vermutlich Fehlanga- ben evoziert aufgrund des erfahrungsgemäss grossen Anteils informeller Tätigkeiten am Gesamteinkommen.

In Abbildung 4 wird letztlich deutlich, dass die «Chruščëvki» nach 1995 vor allem von Haushalten mit geringerem Einkommen in die engere Auswahl bei Wohnstandortentscheidungen genommen wurden, während Wohnungen der $137 \mathrm{er}$ Serie oder der modernen, nach 1995 errichteten vielgeschossigen Gebäude einen höheren Anteil an besserverdienenden Haushalten anzogen. Sowohl im Vergleich mit der Serie 137 und dem neuen Geschosswohnungsbau (Abb. 4), als auch im Vergleich zu den untersuchten innerstädtischen und suburbanen Wohnquartieren, erlebten die «Chruščëvki» damit nach 1990 Tendenzen sozioökonomischer Abwertung: ihnen gelang es nicht, im gleichen Masse mittlere und höhere Einkommensgruppen anzuziehen. Der unerwartet hohe Anteil an besser gestellten Haushalten, die nach 1995 in die Chruščëvki zuzogen, ist dabei auf den nach wie vor sehr angespannten Wohnungsmarkt zurückzuführen, der in allen Preissegmenten Angebotsdefizite aufweist. In vielen Fällen dienen die Wohnungen in den Chruščëvki für Besserverdienende als zeitlich begrenzte Übergangslösung. Der hohe Anteil an Geringverdienenden im neuen Geschosswohnungsbau erklärt sich durch eine Spezifik des Wohnraumerwerbs insbesondere in den 1990er Jahren. Aufgrund des aus Sowjetzeiten übertragenen Rechts jeder Familie auf Wohnraum kann man bis heute über die Kommune eine subventionierte Wohnung beantragen. Mit der kompletten Übergabe des Wohnungsbaus aus der staatlichen bzw. kommunalen Hand an privatisierte Bauunternehmen wurde die Übergabe bestimmter Anteile an neu gebauten Wohnungen (ca. 10-15\%) an die Kommune zu deren freier Verfügung ausgehandelt. Dieses Kontingent wird von der Kommune zu einem Teil an Familien auf der Liste der Wohnungssuchenden vergeben, der andere Teil wird auf dem Wohnungsmarkt angeboten. Aufgrund der sehr langen Wartezeiten (in St. Petersburg je nach Wohnungsgrösse mehr als zehn Jahre) sind auf der Liste fast ausschliesslich Familien mit geringem Einkommen verblieben, die sich zu üblichen Marktbedingungen keine Wohnung kaufen können.

Die Analyse weiterer soziostruktureller Merkmale (Alter, Haushaltsstruktur und Qualifikationsniveau) der nach 1995 in die untersuchten Wohnquartiere der Stadt Zugezogenen lässt Aussagen zu, dass in die «Chruščëvki» zwar ein relativ hoher Anteil an jungen Haushalten (unter 35 Jahre, 63\%) zuzog, diese Haushalte aber sehr häufig von allein erziehenden Eltern geführt waren mit Einkommensverhältnissen (entsprechend der Eigeneinschätzung) im unteren und mittleren Bereich (40\%). Demgegenüber zogen in die Serie 137 vor allem junge Familien mit mehr 


\begin{tabular}{|l|c|c|c|}
\hline Quartier & vor 1990 & $\mathbf{1 9 9 0 - 2 0 0 0}$ & $\mathbf{2 0 0 1 - 2 0 0 7}$ \\
\hline Innerstädtisches Wohngebiet (N=97) & 60 & 19 & 21 \\
\hline Chrušč̈̌ka $(\mathrm{N}=150)$ & 64 & 19 & 17 \\
\hline Serie 137 (N=146) & 70 & 18 & 12 \\
\hline Geschosswohnungsbau nach 1995 (N=146) & 0 & 14 & 86 \\
\hline Suburbanes Einfamilienhausgebiet $(\mathrm{N}=55)$ & 0 & 38 & 62 \\
\hline
\end{tabular}

Tab. 3: Zeitpunkt des Zuzugs der befragten Bewohner in das Quartier (in Prozent)

Date of the respondent's in-migration to the neighbourhood (in percent)

Dates d'arrivée dans les différents quartiers (en pourcents)

Quelle: eigene Erhebung (2007)

als einem Kind und mittlerem Einkommen ein. Der neue Geschosswohnungsbau profitierte vor allem von Eltern mittleren Alters (35-49 Jahre) mit einem Kind, sehr guter Ausbildung (76,3\% mit Hochschulabschluss) und mittlerem Einkommen.

Im Ergebnis der soziostrukturellen Analysen ist festzuhalten, dass sich für die «Chruščëvki» durch den Zuzug nach 1995 vor allem problematische soziale Entwicklungstendenzen andeuten, sie also im intraregionalen Vergleich mit den innerstädtischen und suburbanen Wohnquartieren und im Gegensatz zu der Serie 137 und dem neuen Geschosswohnungsbau Tendenzen einer soziostrukturellen Abwertung erfahren. Für die Serie 137 und den neuen Geschosswohnungsbau zeichnen sich hingegen relativ stabile bzw. leicht positive soziostrukturelle Entwicklungen im stadtregionalen Vergleichskontext ab.

Diese Aussagen zu Tendenzen der soziostrukturellen Auf- und Abwertung bzw. Persistenz sind allerdings nicht gleichzusetzen mit Aussagen zu aktuellen Gesamtbewohnerstrukturen in den untersuchten Grosswohnsiedlungen. Denn wie in Tabelle 3 deutlich wurde, wurden die aus sozialistischer Zeit überkommenen Bewohnerstrukturen der GWS-Typen in unterschiedlichem Masse nach 1990 überprägt. Abbildung 5 zur Selbsteinschätzung des Haushalteinkommens aller befragten Bewohner zeigt letztlich deutlich, dass aktuell sowohl die «Chruščèvki» als auch die Serie 137 und der neue Geschosswohnungsbau Wohnorte der Mittelschicht sind: Der Anteil der mittleren Einkommensgruppen ist jeweils der grösste; die höchsten wie die geringsten Einkommensgruppen sind ebenso in den aktuellen Bewohnerstrukturen vertreten. Lediglich für den neuen Geschosswohnungsbau deutet sich ein vergleichsweise geringerer Anteil einkommensschwächerer Haushalte an. Die Bewohner des neuen Geschosswohnungsbaus fühlen sich zudem am häufigsten als «Gewinner» der Nachwendezeit (Tab. 4).

\section{Fazit: Sozialräumliche Differenzierung oder Polarisierung?}

Der Beitrag verdeutlicht, dass die Grosswohnsiedlungen vor allem Wohnorte der Mittelschicht sind. Sie weisen ein breites Einkommensspektrum auf, sind Wohnorte vor allem von Familien im jüngeren und mittleren Alter, die mit ihrer Wohnsituation überwiegend zufrieden sind. Im Vergleich zur Situation der Grosswohnsiedlungen im Sozialismus kann auf eine soziale Persistenz geschlossen werden. Auch im Sozialismus waren die GWS, die «Chruščëvki» und die Serie 137, vor allem Wohnorte der Mittelschicht. Die GWS standen in der symbolischen Hierarchie der Wohnpräferenzen der Stadtbewohner im Mittelfeld.

Hinsichtlich der symbolischen und soziostrukturellen Position der GWS werden jedoch seit 1990 Veränderungen deutlich. Bei den Wohnpräferenzen erfuhren die sowjetischen GWS relative symbolische Abwertungen im intraregionalen Kontext. Die nach 1995 errichteten Grosswohnsiedlungen stehen in der Wohnpräferenz über den sowjetischen, erleben seit Mitte der 1990er Jahre eine anhaltend grosse Nachfrage vor allem durch besser verdienende Haushalte und sind eine bezahlbare Alternative zum prestigträchtigen Wohnen im Einfamilienhaus im Grünen.

Tendenziell nehmen auch die sozialräumlichen Differenzierungen zwischen den GWS-Typen «Serie 137», «Chruščëvki» und «neuer Geschosswohnungsbau» $\mathrm{zu}$, ohne bisher in sozialräumliche Polarisierungen zu münden. Während die «Serie 137» als «Insel der sozialen Persistenz» bezeichnet werden kann und der «Geschosswohnungsbau nach 1995» sich als Wohnort der «gefühlt» erfolgreichen, jungen Mittelschicht etabliert, zeichnen sich für die «Chruščëvki» sanfte sozioökonomische Abwertungstendenzen ab, die sich in der Zukunft verstärken können. 


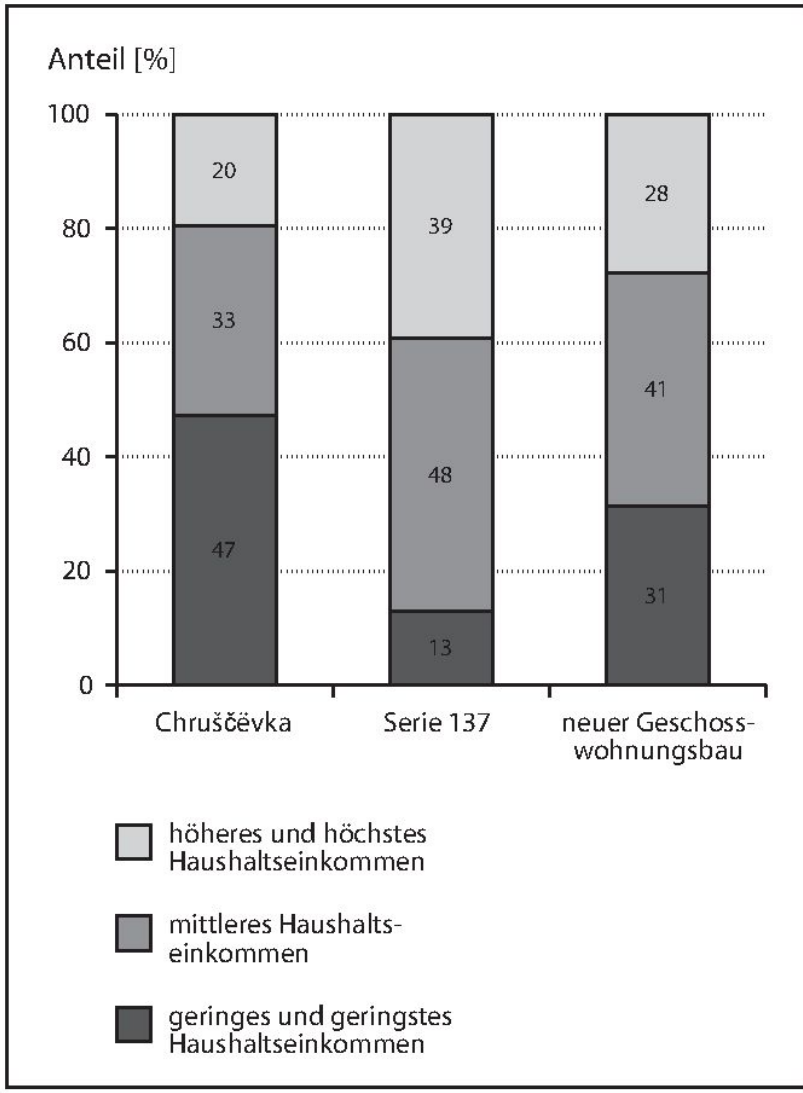

Abb. 4: Einkommensgruppen der nach 1998 in die Wohnquartiere Zugezogenen

The income of households who moved to the respective neighbourhoods after 1998

Revenu des ménages installés dans les quartiers après 1998

Quelle: eigene Erhebung (2008); Entwurf: Projekt MOE Wohnen; Graphik: A. MüLleR

Bisher schlagen sich diese symbolischen und soziostrukturell differenzierenden Entwicklungstendenzen nach 1990 jedoch noch nicht vollends im Sozialraum der Stadt nieder: Die symbolische, relative Abwertung der sowjetischen Grosswohnsiedlungen hat bisher weder $\mathrm{zu}$ einer erhöhten Wohnmobilität, noch zu einer nachlassenden Nachfrage oder zu einem Preisrückgang bei Wohnungen aus der sowjetischen Bauperiode geführt. Mittelfristig wird das auch nicht erwartet, da Alternativen nur sehr eingeschränkt, vornehmlich im höheren Preissegment, zugänglich sind. Langfristig kann jedoch bei anhaltender ökonomischer Konsolidierung und Stabilität, bei Abbau des Angebotdefizits und bei Erweiterung der Alternativen für Wohnentscheidungen eine verstärkte Wohnmobilität erwartet werden, insbesondere innerhalb der Gruppe der Besserverdienenden,

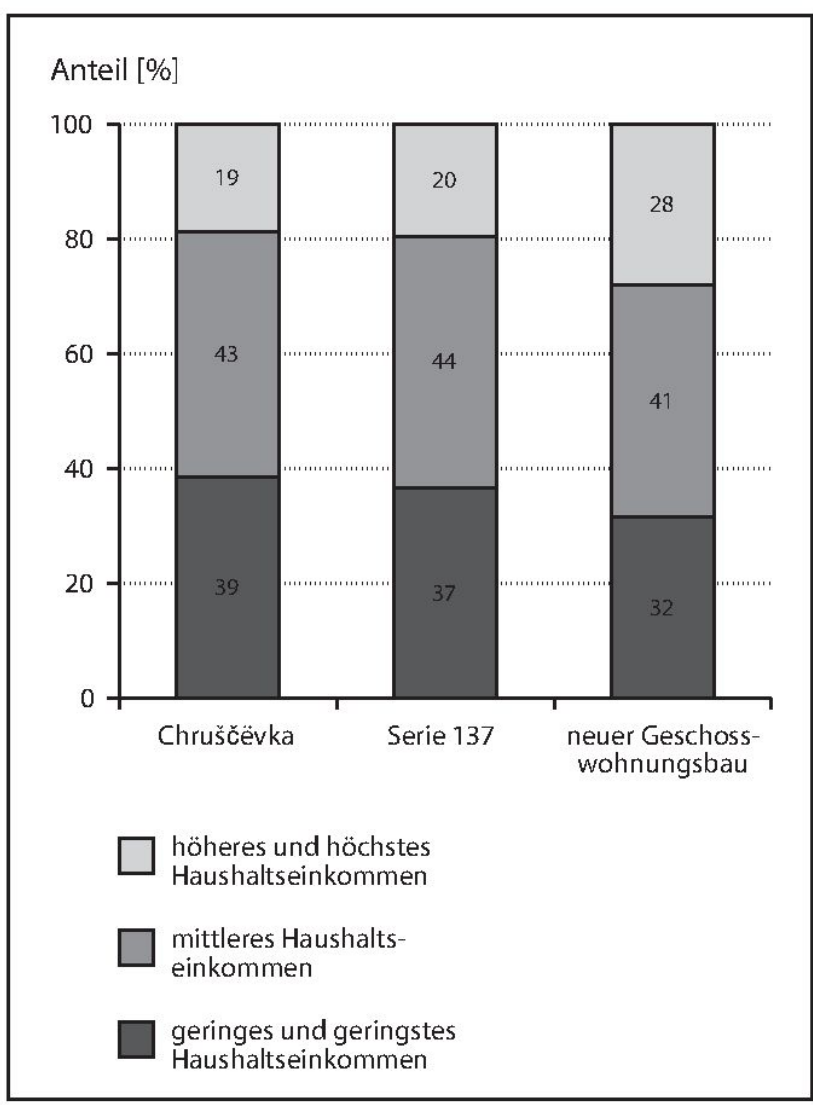

Abb. 5: Anteil der Einkommensgruppen im Jahr 2007, nach Wohnquartierstypen

Percentages of income groups in 2007 differentiated by neighbourhood types

Proportion des catégories de revenus selon les types de quartiers en 2007

Quelle: eigene Erhebung (2007); Entwurf: Projekt MOE Wohnen; Graphik: A. MüLLER

sowie eine verringerte Nachfrage und eine weitere Verschlechterung des Images der Wohnquartiere aus sowjetischer Bauzeit. Für die neu errichteten Siedlungen ist mittelfristig nicht mit einer sozialen Abwertung zu rechnen.

Die sozialräumlichen Differenzierungen werden künftig zwischen den neuen und alten Typen der Grosswohnsiedlungen sowie zwischen den Grosswohnsiedlungen und den anderen städtischen Wohnquartieren vermutlich weiter zunehmen.

\section{Dank}

Die Forschung wurde unterstützt von der Deutschen Forschungsgemeinschaft (Sozialräumliche Differenzierung in MOE-Stadtregionen 2007-2010). 


\begin{tabular}{|l|c|c|c|c|c|c|}
\hline & \multicolumn{2}{|l|}{ Lebenssituation } & \multicolumn{2}{l|}{ Berufliche Situation } \\
\cline { 2 - 7 } & Chruščëvka & Serie 137 & $\begin{array}{l}\text { Geschoss- } \\
\text { wohnungs- } \\
\text { bau nach } \\
1995\end{array}$ & Chruščëvka & Serie 137 & $\begin{array}{l}\text { Geschoss- } \\
\text { wohnungsbau } \\
\text { nach 1995 }\end{array}$ \\
\hline $\begin{array}{l}\text { stark verbessert/ } \\
\text { eher verbessert }\end{array}$ & 47,3 & 58,1 & 65,3 & 48,0 & 50,4 & 64,6 \\
\hline $\begin{array}{l}\text { stark } \\
\text { verschlechtert/ } \\
\begin{array}{l}\text { eher } \\
\text { verschlechtert }\end{array}\end{array}$ & 18,2 & 18,9 & 11,1 & 14,2 & 11,9 & 8,4 \\
\hline
\end{tabular}

Tab. 4: «Hat sich in den letzten 15 Jahren Ihre berufliche Situation sowie die Zufriedenheit mit Ihrer Lebenssituation insgesamt eher verbessert oder verschlechtert?» (in Prozent)

«Did your professional situation and your satisfaction with your living situation improve on the whole or worsen during the last 15 years?» (in percent)

«Votre situation professionnelle et votre satisfaction résidentielle se sont-elles améliorées ou dégradées au cours des 15 dernières années?» (en pourcents)

Quelle: eigene Erhebung (2007)

\section{Literatur}

AksËnov, K. (2008): Tajny izbiratel'nogo Bjulletenja. Ėlektoral'nye buri i štili severnoj stolicy 1989-2004. Izd-vo S.-Peterburgskogo gosudarstvennogo universiteta.

Axenov, K., Brade, I. \& E. Bondarchuk (2006): The transformation of urban space in post-Soviet Russia. London: Routledge.

Beckhoven, E. \& R. van Kempen (2006): Towards more social cohesion in large post-Second World War housing estates? - In: Housing Studies 21, 4: 477-500.

Bernt, M. \& S. Kabisch (2006): Ostdeutsche Grosswohnsiedlungen zwischen Stabilisierung und Niedergang. - In: DISP 164, 1: 5-15.

Brade, I., Herfert, G. \& K. Wiest (2009): Recent trends and future prospects of socio spatial differentiation in urban regions of Central and Eastern Europe: A lull before the storm? - In: Cities 26, 5: 233-244.

Burdack, J. \& R. RudolPh (2001): Postsozialistische Stadtentwicklungen zwischen nachholender Modernisierung und eigenem Weg. - In: Geographica Helvetica $56,4: 261-273$.

Burmeister, J. (2009): Leben neben der Abrissbirne. - = Wissenschaftliche Beiträge, Reihe Sozialwissenschaften 18, Marburg: Tectum-Verlag.

Divinsky, B. (2004): Slovakia - A continuing role for high-rise housing estates. - In: Turkington, R., van KEMPEN, R. \& F. WASSENBERG (eds): High-rise housing in Europe. $-=$ Housing and Urban Policy Studies 28, Delft: 215-230.

Durmanov, V. \& D. Dubbeling (2004): Ukraine - inheritance of centralised planning. - In: TURKINGTON, R., van Kempen, R. \& F. Wassenberg (eds): High-rise housing in Europe. $-=$ Housing and Urban Policy Studies 28, Delft: 203-214.

Hagen-Demszky, A. von der (2009): Reichenfeld Wohnfeld der Reichen? Geschichte und Alltag einer Plattenbausiedlung in Budapest. - In: BoHN, T. (Hrsg.): Von der «europäischen Stadt» zur «sozialistischen Stadt» und zurück? - Bad Wiesseer Tagungen des Collegium Carolinum 29, München: 419-434.

HIRT, S. (2008): Landscapes of postmodernity. Changes in the built fabric of Belgrade and Sofia since the end of socialism. - In: Urban Geography 29, 8: 785810.

KAHL, A. (2003): Erlebnis Plattenbau. Eine Langzeitstudie. - Opladen: Leske + Budrich.

Kabisch, S. \& K. Grossmann (2010): Einwohnerbefragung im Rahmen der Intervallstudie «Leben und Wohnen in Leipzig-Grünau 2009. Ergebnisbericht».http://www.leipzig.de/gruenau 30.06.2010.

KICINSKI, A. (2004): Poland - a future for the «blokowisko»? - In: Turkington, R., van Kempen, R. \& F. WASSENBERG (eds): High-rise housing in Europe. - = Housing and Urban Policy Studies 28, Delft: 187-202.

Kovács, Z. \& M. Douglas (2004): Hungary - from socialist ideology to market reality. - In: TuRKINGTON, R., van Kempen, R. \& F. WASSEnBerg (eds): High-rise housing in Europe. $-=$ Housing and Urban Policy Studies 28, Delft: 231-248.

Lenkeviciute, J. (2006): Vilnius im Wandel. Wohnsegregation in einer ostmitteleuropäischen Hauptstadt. -Wissenschaftlicher Verlag Berlin. 
Liebmann, H. (2006): Genese, Stand und Perspektiven der Großsiedlungen in Ostmitteleuropa. - In: Informationen zur Raumentwicklung 2006/3-4: 215224.

MilsteAd, T. (2008): Housing and urban development in a post-soviet city: a case study of Vilnius. - http:/ etd.lib.fsu.edu/theses/available/etd-03242008-164148/ unrestricted/MilsteadTSpring2008.pdf 20.9.2010.

Musterd, S. \& R. van Kempen (2007): Trapped or on the springboard? Housing careers in large housing estates in European cities. - In: Journal of Urban Affairs 29, 3: 311-329.

Neugebauer, C., Wiest, K. \& D. Krupickaite (2011): Zukunftsperspektiven mittel- und osteuropäischer Grosswohnsiedlungen zwischen Wohnungsmarkt, Bewohnerinitiative und Förderpolitik. - In: Raumforschung und Raumordnung, Online First, 3. Feb. 2011: http:/www.springerlink.com/content/0034-0111 08.02.2011.

Predloženija po koordinacii Genplana i kompleksnogo plana ékonomičeskogo i socialnogo razvitija Leningrada i oblasti (1980). - = Naučno-techničeskij otčet, Leningrad: LenNIIProekt.

Restate (2005): Project within the Fifth Framework Programme of the EU, «Energy, Environment and Sustainable Development». - http://www.restate.geog. uu.nl 11.07.2010.

Rudolph, R. \& K. Axenov (2003): St. Petersburg postsowjetische Aufwertung von Stadtquartieren. - In: Geographische Rundschau 55, 12: 42- 48.

SankT-Peterburg 1703-2003 (2004): Jubilejnyj statističeskij sbornik. - S.-Peterburg: Sudostroenie. SAnKt-Peterburg 2005 (2006): Statističeskij sbornik. S.-Peterburg: Petrostat.

Spravočnik o domovom fonde Leningrada (1990): Spravočnik o domovom fonde Leningrada. - Leningrad: Proizvodstvennoe ob"edinenie žiliščnogo chozjajstva ispolkoma Lensoveta.

SteInfüHrer, A. (2001): Wandel und Persistenz innerstädtischer Segregation in Ostmitteleuropa. Beispiele aus Brno, Tschechische Republik. - In: Europa Regional 12, 4: 212-222.

Szelenyi, I. (1996): Cities under socialism - and after. - In: Andrusz, G. (ed.): Cities after socialism. Urban and regional change and conflict in post-socialist cities. - Oxford: Blackwell: 286-317.

Tsenkova, S. (2006): Beyond transitions: Understanding urban change in post-socialist cities. - In: TsENKova, S. \& Z. Nedovic-Budic (eds): The urban mosaic of post-socialist Europe. - Heidelberg: Physica-Verlag: 21-50.

Turkington, R., van Kempen, R. \& F. WassenberG (eds) (2004): High-rise housing in Europe: current trends and future prospects. $-=$ Housing and Urban Policy Studies 28, Delft.

Van Kempen, R., Dekker, K., Hall, S. \& I. Tosics (2005): Restructuring large housing estates in Europe.
Restructuring and resistance inside the welfare industry. - Bristol: Policy Press.

WASSENBERG, F. (2004): Large social housing estates: From stigma to demolition? - In: Journal of Housing and the Built Environment 19: 223-232.

\section{Zusammenfassung: Grosswohnsiedlungen in St. Petersburg zwischen sozialräumlicher Polarisierung und Persistenz}

Nach wie vor prägen Grosswohnsiedlungen wesentlich das Bild der postsozialistischen Stadt, da sie im städtischen Raum hinsichtlich ihrer Einwohnerzahl und flächenmässigen Ausmasse, aber auch aufgrund ihrer gesellschaftlichen Wahrnehmung und ihrer Akzeptanz durch die Bewohner ein besonderes Phänomen darstellen. Während sie vor allem in den mitteleuropäischen Ländern zu sozialen Problemgebieten stigmatisiert werden, werden sie in den Städten des östlichen Europa sehr viel differenzierter wahrgenommen.

Die Ergebnisse einer Untersuchung in der Stadtregion St. Petersburg zeigen, dass mit zunehmenden Wohnalternativen in den letzten Jahren eine verstärkte sozialräumliche Differenzierung innerhalb der Grosswohnsiedlungstypen zu beobachten ist, dass die Akzeptanz und Nachfrage danach - differenziert nach Wohnquartierstypen - anhaltend ist. Die untersuchten Wohnquartiere repräsentieren drei bauzeitlich und morphologisch unterschiedliche Grosswohnsiedlungstypen in St. Petersburg.

Schlüsselwörter: Grosswohnsiedlung, sozialräumliche Differenzierung, Persistenz, Polarisierung, St. Petersburg

\section{Abstract: Large scale housing estates in St. Petersburg between socio-spatial polarisation and persistency}

Large scale housing estates still shape the image of the post-socialist cities: they represent a particular phenomenon due to their size in terms of space and population, as well as due to their societal perception and acceptance by their inhabitants and city dwellers in general. While stigmatised as socially problematic neighbourhoods in Western Europe, their perception in Eastern European urban regions is much more differentiated.

The results of an urban regional study about St. Petersburg reveal that - along with the growing differentiation of local housing opportunities - the socio-spatial differentiation among the various types of large scale housing estates increases. Meanwhile, the acceptance and demand for them is stable. The paper investigates three types of large scale housing estates in St. Petersburg that differ in morphology and date of construction. 
Keywords: large scale housing estates, socio-spatial differentiation, persistency, polarisation, St. Petersburg

\section{Résumé: Les grands ensembles à Saint-Pétersbourg entre polarisation socio-spatiale et stabilité}

Les grands ensembles marquent toujours l'image de la ville post-socialiste. Ils constituent un phénomène singulier, non seulement en raison de leur importance démographique et de leur empreinte dans la ville, mais également du fait de la perception et du sentiment d'appartenance de leurs habitants et des citadins en général. Bien que stigmatisés comme des quartiers à problèmes sociaux en Europe occidentale, leur perception dans les régions urbaines d'Europe de l'Est est plus nuancée.

Cet article présente les résultats d'une étude conduite dans la région urbaine de Saint-Pétersbourg dans trois grands ensembles à la morphologie et à la date de construction différentes. Il montre qu'une différenciation socio-spatiale entre les grands ensembles opère parallèlement à une différenciation entre opportunités résidentielles locales. L'acceptation de ces grands ensembles et leur demande reste stable dans le temps.
Mots-clés: grands ensembles, ségrégation socio-spatiale, persistance, polarisation, Saint-Pétersbourg

Dr. Isolde Brade, Dipl.-Ing. Carola Silvia Neugebauer, Leibniz-Institut für Länderkunde, Schongauerstrasse 9, D-04329 Leipzig, Deutschland.

e-mail:

I_Brade@ifl-leipzig.de

C_Neugebauer@ifl-leipzig.de

Dr. Konstantin Axenov, Associate Professor, Department of Regional Diagnostics and Political Geography, St. Petersburg State University, 10-th Linia V.O., 33, 199178 St. Petersburg, Russian Federation.

e-mail:poaxenov@peterlink.ru

Manuskripteingang/received/manuscrit reçu le 26.10.2010

Annahme zum Druck/accepted for publication/accepté pour publication: 29.3 .2011 